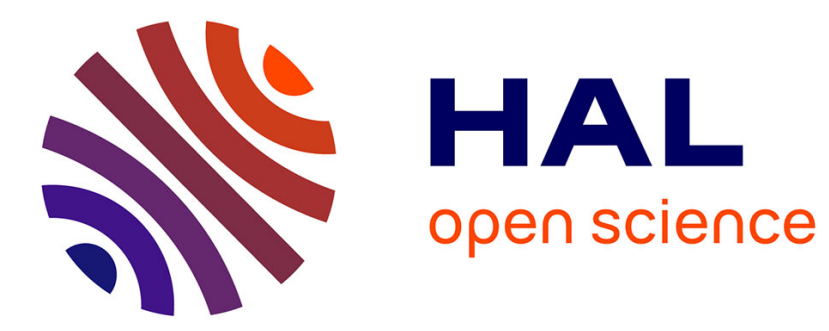

\title{
Recent Improvements for Solving Inverse Magnetostatic Problem Applied to Thin Shells
}

Olivier Chadebec, Jean-Paul Bongiraud, Gilles Cauffet, Philippe Le Thiec

\section{To cite this version:}

Olivier Chadebec, Jean-Paul Bongiraud, Gilles Cauffet, Philippe Le Thiec. Recent Improvements for Solving Inverse Magnetostatic Problem Applied to Thin Shells. IEEE Transactions on Magnetics, 2002, 38 (2), pp.1005-1008. 10.1109/20.996258 . hal-00289969

\section{HAL Id: hal-00289969 https://hal.science/hal-00289969}

Submitted on 24 Jun 2008

HAL is a multi-disciplinary open access archive for the deposit and dissemination of scientific research documents, whether they are published or not. The documents may come from teaching and research institutions in France or abroad, or from public or private research centers.
L'archive ouverte pluridisciplinaire HAL, est destinée au dépôt et à la diffusion de documents scientifiques de niveau recherche, publiés ou non, émanant des établissements d'enseignement et de recherche français ou étrangers, des laboratoires publics ou privés. 


\title{
Recent Improvements for Solving Inverse Magnetostatic Problem Applied to Thin Shells
}

\author{
Olivier Chadebec, Jean-Louis Coulomb, Jean-Paul Bongiraud, Gilles Cauffet, and Philippe Le Thiec
}

\begin{abstract}
In this paper, we propose a new approach to solve the magnetostatic inverse problem. The goal of the work is, from measurements of the magnetic field in the air, to rebuild a model for the magnetization of a ferromagnetic shell structure. It's then possible to calculate the field where sensors cannot be placed. This problem is usually ill posed or rank-deficient, it's then necessary to use mathematical regularizations. These techniques are based upon the injection of knowledge about the mathematical behavior of the solution. We preferred to add physical information. This solution allows us to get a faithful solution and to reduce significantly the number of sensors. Moreover, our method has been tested on a mock-up with real measurements and led to very promising results.
\end{abstract}

Index Terms-Inverse problem, magnetization identification, magnetostatic, moments method, thin shell.

\section{INTRODUCTION}

$\mathbf{M}$ ORE AND MORE applications nowadays deal with inverse problems. In this paper, we are interested by the inverse magnetostatic problem. A ferromagnetic shell with an unknown magnetization, placed in an external inductor field is considered. The total magnetization of the sheet can be divided in two parts: 1) an induced one, due to the reversible reaction of the material to the inductor field and 2) a remanent one due to the magnetic history of the material (which depends on hysteresis, mechanical, and thermal constraints).

The calculus of the induced magnetization is now well-known [1], [2]. In a first step, we propose a new formulation to calculate this magnetization.

However, the remanent one is impossible to evaluate with a deterministic calculus, because, generally, we have no access to the magnetic past of the material. Moreover, even if we had such a knowledge, existing models would be too complex to apply to three-dimensional (3-D) geometries. It's then necessary to use magnetic measurements to determine the total magnetization of the shell. The main goal of this work is to solve an inverse problem (i.e., determination of the sources by knowing the effect) with magnetic sensors placed in the air region. The relation between the distribution of the magnetization and the measurements leads to a linear system, but its resolution is not trivial and the main difficulty comes from the lack of unicity of

Manuscript received July 5, 2001; revised October 25, 2001. This work is supported by the French Navy (DGA-GESMA).

O. Chadebec, J.-P. Bongiraud, G. Cauffet, and P. Le Thiec are with the Laboratoire du Magnétisme du Navire, ENSIEG, Saint Martin d'Hères 38402, France (e-mail: chadebec@leg.ensieg.inpg.fr).

J.-L. Coulomb is with the Laboratoire d'Electrotechnique de Grenoble, INPG/UJF CNRS UMR 5529, ENSIEG, St. Martin d'Hères 38402, France (e-mail: coulomb@leg.ensieg.inpg.fr).

Publisher Item Identifier S 0018-9464(02)02443-3. the solution. Usually, to overcome this difficulty, regularization techniques are used. However, we will see that these approaches are very difficult to apply to 3-D thin shells geometries.

Then, we propose a new method which avoids using classical regularization, injecting physical knowledge about the magnetic behavior of the sources. By changing the magnetic material law used for the calculus of the induced magnetization, it is possible to take into account the remanent one. We obtain then a linear system valid inside the shell. This system is then associated with the linear system associated to the measurement, and the global resolution gives a unique solution. This better knowledge of the behavior of the material allows then to use less sensors. Once the total magnetization is determined, it is easy to calculate the field everywhere in the air region, in particular, where sensors cannot be placed.

In this paper, we are especially interested in the magnetic anomaly created by ferromagnetic ships. Our approach will make it possible to predict the offboard magnetic anomaly created by such ships with onboard measurements.

\section{CAlculus OF the InduCEd Magnetization}

\section{A. An Equation for the Material}

In this section, we are interested in the calculus of induced magnetization $\mathbf{M}^{\text {ind }}$, knowing the external field $\mathbf{H}_{\mathbf{0}}$, the geometry and the reversible permeability $\mu$ of the material. This kind of calculus is a typical direct problem (determination of the effect by knowing sources) and is well known. However, we propose a new formulation more adapted to solve the inverse problem.

Consider a ferromagnetic shell placed in a static external inductor field $\mathbf{H}_{\mathbf{0}}$ (earth magnetic field or field created by coils). The induced magnetization of the shell creates a local perturbation of the field, which can be expressed in terms of reduced field $\mathbf{H}_{\text {red }}$

$$
\mathbf{H}=\mathbf{H}_{\mathbf{0}}+\mathbf{H}_{\text {red }} .
$$

An equation representative of a reversible variation of the induced magnetization in low field is

$$
\mathbf{B}=\mu \mathbf{H}
$$

where $\mu$ is the constant reversible permeability of the material. It must be pointed out that this material law only takes into account reversible variation of magnetization with respect to a remanent magnetization equal to zero. Now consider the following general equation:

$$
\mathbf{B}=\mu_{0}\left(\mathbf{H}+\mathbf{M}^{\text {ind }}\right) .
$$


By combining (1)-(3), we obtain a local internal equation for the sheet

$$
\mathbf{M}^{\text {ind }}=\left(\mu_{r}-1\right)\left(\mathbf{H}_{\text {red }}+\mathbf{H}_{\mathbf{0}}\right)
$$

where $\mu_{r}$ is the relative reversible permeability and $\mathbf{H}_{\text {red }}$ is the field at one point of the shell and is created by the magnetization of all the sheet.

\section{B. Reduction of the Singularity}

The relation between $\mathbf{H}_{\text {red }}$ in the air region and $\mathbf{M}^{\text {ind }}$ is then given by [3]

$$
\mathbf{H}_{\text {red }}=\frac{1}{4 \pi} \iiint_{V} \frac{\left(3\left(\mathbf{M}^{\text {ind }} \cdot \mathbf{r}\right) \mathbf{r}-r^{2} \mathbf{M}^{\text {ind }}\right.}{r^{5}} d V
$$

where $r$ is the vector between a point of the shell and the integration point of the shell.

If the thickness $e$ of the sheet is small and relative permeability $\mu_{r}$ high, we assume that this magnetization is tangential to $S$, the median surface [1]. The shell is meshed into $N$ elements with a uniform magnetization affected on each of them. By combining (4) and (5) and applying a collocation method at the barycenter of element $j$, we obtain

$$
\begin{aligned}
\mathbf{M}_{\mathbf{j}}^{\text {ind }}=\left(\mu_{r}-1\right) & \left(\mathbf{H}_{\mathbf{0}}+\frac{e}{4 \pi} \sum_{i}^{N}\right. \\
& \left.\cdot \iint_{S_{i}} \frac{\left(3\left(\mathbf{M}_{\mathbf{i}}^{\text {ind }} \cdot \mathbf{r}\right) \mathbf{r}-r^{2} \mathbf{M}_{\mathbf{i}}^{\mathbf{i n d}}\right.}{r^{5}} d S_{i}\right) .
\end{aligned}
$$

Equation (6) presents the drawback to be singular for $i=j$ with an order of $1 / r^{3}$. To reduce this singularity, we are going to apply a classical result on each element. Considering a volume $V$ with a uniform magnetization, we have

$$
\begin{aligned}
\mathbf{H}_{\text {red }} & =\frac{1}{4 \pi} \iiint_{V} \frac{\left(3(\mathbf{M} \cdot \mathbf{r}) \mathbf{r}-r^{2} \mathbf{M}\right.}{r^{5}} d V \\
& =-\frac{1}{4 \pi} \iint_{S} \mathbf{M} \cdot \mathbf{n} \frac{\mathbf{r}}{r^{3}} d S
\end{aligned}
$$

where $\mathbf{n}$ is the external normal to $V$. By applying (4) on each element $S_{i}$ and by reducing it to a shell element, we obtain a new expression for (6)

$$
\mathbf{M}_{\mathbf{j}}^{\mathbf{i n d}}=\left(\mu_{r}-1\right)\left(\mathbf{H}_{\mathbf{0}}-\frac{e}{4 \pi} \sum_{i}^{N} \int_{L_{i}} \mathbf{M}_{\mathbf{i}}^{\mathbf{i n d}} \cdot \mathbf{n}_{\mathbf{i}} \frac{\mathbf{r}}{r^{3}} d L_{i}\right)
$$

where $\mathbf{n}_{\mathbf{i}}$ is an external normal to the thickness of element $S_{i}$.

Equation (8) does not present any singularity because the collocation point is at the barycenter of the element and the integration points are located on edges of the mesh.

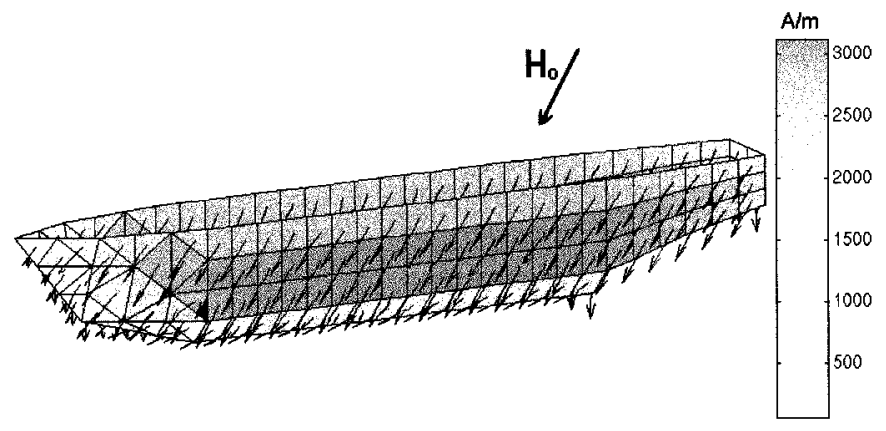

Fig. 1. Example of induced magnetization created by an external field of $46000 \mathrm{nT}$ on a mock-up of a ship ( $4.60 \mathrm{~m}$ long, $\left.\mu_{r}=96, e=1.4 \mathrm{~mm}\right)$.

\section{Resolution of the System}

By writing (8) on each element, we obtain a full square system of $2 \times N$ equations (two components of the magnetization per element)

$$
\left[\mathbf{C}+\mathbf{I}_{\mathbf{d}}\right]\left[\mathbf{M}^{\text {ind }}\right]=[\mathbf{d}]
$$

where $\mathbf{I}_{\mathbf{d}}$ is the identity matrix and $\mathbf{d}$ depends on the inductor field and on the relative reversible permeability. This system has a good condition number and can be inverted with classical approach (single value decomposition, for example). This resolution leads the to induced magnetization (see Fig. 1).

It is then easy to calculate the induction everywhere in the air region with the following equation:

$$
\mathbf{B}=\mu_{0}\left(\mathbf{H}_{\mathbf{0}}-\frac{e}{4 \pi} \sum_{i}^{N} \int_{L_{i}} \mathbf{M}_{\mathbf{i}}^{\mathbf{i n d}} \cdot \mathbf{n}_{\mathbf{i}} \frac{\mathbf{r}}{r^{3}} d L_{i}\right) .
$$

The induction is calculated with an order of $1 / r^{2}$, which leads to a better accuracy of the calculated field close to the shell (where we are going to place the sensors). Moreover, integrations are now realized on line elements and the constant magnetization distribution on each element is mathematically equivalent to line charge distribution on the edges of the mesh. We will use (9) to solve the inverse problem.

\section{RESOLUTION OF THE INVERSE PROBLEM}

\section{A. Classical Approach}

We developed a new formulation to compute the induced magnetization of ferromagnetic shell. However, our problem is more complex. Basically, as we said, the total magnetization of the sheet is composed of a induced one $\mathbf{M}^{\text {ind }}$ and a remanent one $\mathbf{M}^{\mathrm{rem}}$. It is then necessary to use magnetic measurements to determine $\mathbf{M}$. Let us consider a magnetic triaxial sensor located in the air, close to the shell. The relation which links the measured field to $\mathbf{M}$ is

$$
\mathbf{B}_{\text {sensor }}=\mu_{0}\left(\mathbf{H}_{\mathbf{0}}-\frac{e}{4 \pi} \sum_{i}^{N} \int_{L_{i}} \mathbf{M}_{\mathbf{i}} \cdot \mathbf{n}_{\mathbf{i}} \frac{\mathbf{r}}{r^{3}} d L_{i}\right) .
$$

Placing $P$ sensors in the air region. We can then write a system of $2 \times N$ unknowns and $3 \times P$ equations. We obtain the following system:

$$
[\mathbf{A}][\mathbf{M}]=[\mathrm{b}]
$$


where $\mathbf{A}$ depends on sensor positions, on the mesh and where the components of $\mathrm{b}$ are the measured reduced fields. Classically, solving the magnetostatic inverse problem consist in finding a solution for only system (11) [4].

\section{B. Difficulties for Solving Inverse Problems}

Two kinds of difficulties can be associated to inverse problems. Generally, for most part of applications, lots of measurements are taken to obtain more equations than unknowns. The system obtained is then very large (especially for 3-D geometries) and some equations are close to the contradiction (due to the inaccuracies of the measurements). The system has then a very bad condition number and the solution is divergent due to the noise of measurements. It is said ill posed. Now, if there are only few sensors, equations could not be contradictory, but the system is rank deficient (less equation than unknowns). Then, several distributions are going to fit the measurements. In both cases, the solution is not unique. To solve an inverse problem consist in selecting the good one.

Classical approach is then to use Tikhonov's regularization [5]. This method is based on the addition of a known information. It is then necessary to solve (11), plus one new mathematical system weighted by a small coefficient. Instead of finding M such as

$$
\text { M minimizes }\|\mathbf{A M}-\mathbf{b}\| \text {. }
$$

The goal is to find $\mathbf{M}$ such as

$$
\mathbf{M} \text { minimizes }\|\mathbf{A M}-\mathbf{b}\|+\alpha\|\mathbf{L M}\| \text {. }
$$

The choice of the matrix $\mathbf{L}$ depends on which kind of information we want to add. If $\mathbf{L}$ equal $\mathbf{I}_{\mathbf{d}}$ (i.e., the identity matrix), the method tries to fit the distribution to the magnetic measurements by keeping a minimal norm for $\mathbf{M}$ (0-order regularization). $\mathbf{L}$ can also be chosen as the derivative matrix (first-order regularization), the obtained solution is then the most regular.

In a first step, we tried to use a zeroth-order regularization $\left(\mathbf{L}=\mathbf{I}_{\mathbf{d}}\right), \mathbf{L}$ being easy to compute. However, the approach was not satisfying. Basically, the minimal norm solution is not acceptable for the magnetization. It seemed better to use first-order regularization. However, these approaches are difficult to apply to realistic 3-D thin shell problems. The geometrical complexity of our device and our basis of vectors makes the derivative matrix $\mathbf{L}$ very difficult to compute. Classical regularization method failed to solve the magnetostatic problem applied to thin shell.

\section{EQUATION VALID INSIDE THE SHELL}

The main reproach which could be addressed to regularization method is to choose the solution by taking into account a mathematical criteria. Instead of adding a mathematical information, we tried to add our physical knowledge of the problem.

In (2), we only took into account induced magnetization. In fact, it is possible to add in a model of material both induced and remanent magnetization. The magnetic material law becomes

$$
\mathbf{B}=\mu \mathbf{H}+\mathbf{B}^{\text {rem }} .
$$

Now consider the equivalent of (3):

$$
\mathbf{B}=\mu_{0}\left(\mathbf{H}+\mathbf{M}^{\text {ind }}+\mathbf{M}^{\text {rem }}\right) .
$$

Equation (4) is still valid, but now $\mathbf{H}_{\text {red }}$ is created by $\mathbf{M}^{\text {ind }}$ and $\mathbf{M}^{\mathrm{rem}}$. By an equivalent calculus of the Section II, we obtain a collocation equation for each element of the mesh

$$
\begin{aligned}
\mathbf{M}_{\mathbf{j}}^{\mathbf{i n d}}=\left(\mu_{r}-1\right)\left(\mathbf{H}_{\mathbf{0}}-\frac{e}{4 \pi} \sum_{i}^{N} \int_{L_{i}} \mathbf{M}_{\mathbf{i}}^{\mathbf{i n d}} \cdot \mathbf{n}_{\mathbf{i}} \frac{\mathbf{r}}{r^{3}} d L_{i}\right. \\
\left.-\frac{e}{4 \pi} \sum_{i}^{N} \int_{L_{i}} \mathbf{M}_{\mathbf{i}}^{\mathbf{r e m}} \cdot \mathbf{n}_{\mathbf{i}} \frac{\mathbf{r}}{r^{3}} d L_{i}\right) .
\end{aligned}
$$

By separating $\mathbf{M}^{\text {ind }}$ and $\mathbf{M}^{\text {rem }}$, we obtain a new system representative of the behavior of the shell and its sources

$$
\left[\begin{array}{ll}
\mathbf{C}+\mathbf{I}_{\mathrm{d}} & \mathbf{C}
\end{array}\right]\left[\begin{array}{c}
\mathbf{M}^{\text {ind }} \\
\mathbf{M}^{\text {rem }}
\end{array}\right]=[\mathrm{d}] .
$$

System (15) is composed of $2 \times N$ equations and $4 \times N$ unknowns. It is rank deficient, so its solution is nonunique. This situation could be predicted because we have not added knowledge about the magnetic history. Then, lots of distributions of magnetization are possible. The new system minimize the global energy by taking into account the inductor field $\mathbf{H}_{\mathbf{0}}$ and the behavior of the sources between themselves. Notice that solving (15) with a single value decomposition leads to the calculus of induced magnetization (i.e., created by $\mathbf{H}_{\mathbf{0}}$ ) and force remanent magnetization to zero.

Then, it remains to solve both systems (11) and (15) in a global one to constrain system (15) by measurements. The system obtained is then

$$
\left[\begin{array}{cc}
\mathbf{C}+\mathbf{I}_{\mathbf{d}} & \mathbf{C} \\
\mathbf{A} & \mathbf{A}
\end{array}\right]\left[\begin{array}{c}
\mathbf{M}^{\text {ind }} \\
\mathbf{M}^{\text {rem }}
\end{array}\right]=\left[\begin{array}{l}
\mathbf{d} \\
\mathbf{b}
\end{array}\right] .
$$

Generally, the contradictory equations come from (11) and the larger (11) is, the worse the condition number is. With (16), it is then useless to use a great number of sensors, a large part of the behavior of the sources being well known. This system will have a good condition number and a simple single value decomposition leads generally to a correct solution for the magnetization. The computation of a regularization matrix $\mathbf{L}$ is then useless and the difficulty associated to the choice of the weighted parameter $\alpha$ useless too [6].

\section{NuMERICAL AND EXPERIMENTAL RESUltS}

We are especially interested in the magnetic anomaly created by ferromagnetic ships. We have built a real mockup of a ship (4.60 meter long, $e=1.4 \mathrm{~mm}$ and $\mu_{r}=96$ ) on which we have tested our algorithm. The external magnetic field $\mathbf{H}_{\mathbf{0}}$ is the earth's magnetic field $(46000 \mathrm{nT})$. Thirty-two triaxial fluxgate sensors have been placed inside the ship, which is meshed into 271 elements (see Fig. 2). From measurements taken inside the hull and by using our approach, we obtain the following condition number $\xi$ :

$$
\xi=13.02 .
$$




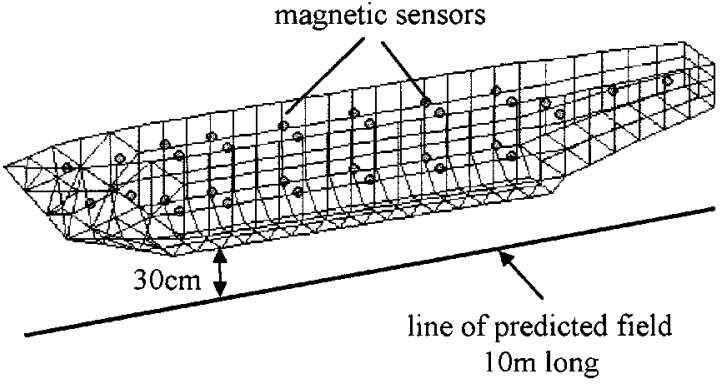

Fig. 2. Mesh of the shell, sensors location and line on which the field is predicted by inverse problem and compared to measurements.

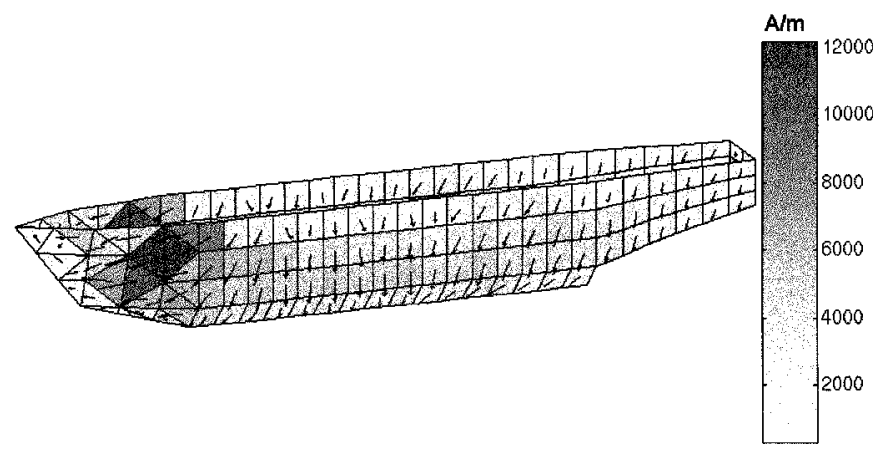

Fig. 3. Model of the magnetization determined by measurements done inside the mock-up.

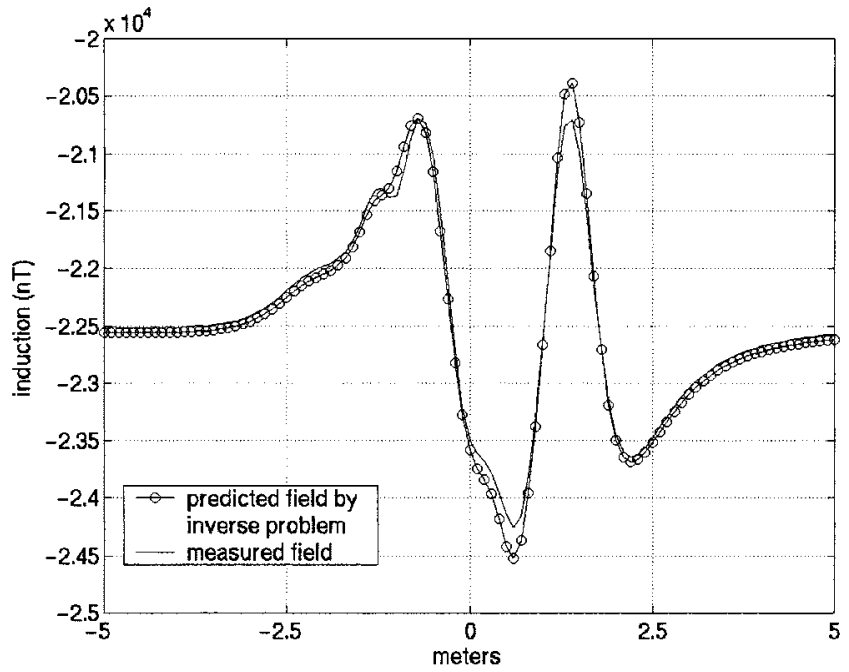

Fig. 4. Comparison of field predicted by inverse problem and measured field. Longitudinal component of the induction outside the ship ( $30 \mathrm{~cm}$ under the hull).

This condition number is good. We can solve this system by a simple single value decomposition and obtain a model of the magnetization (see Fig. 3), allowing to calculate the field outside. The field obtained is then compared to measurements taken on a longitudinal line (10 m long) located $30 \mathrm{~cm}$ under the hull (see Fig. 4 for the longitudinal component and Fig. 5 for the vertical component).

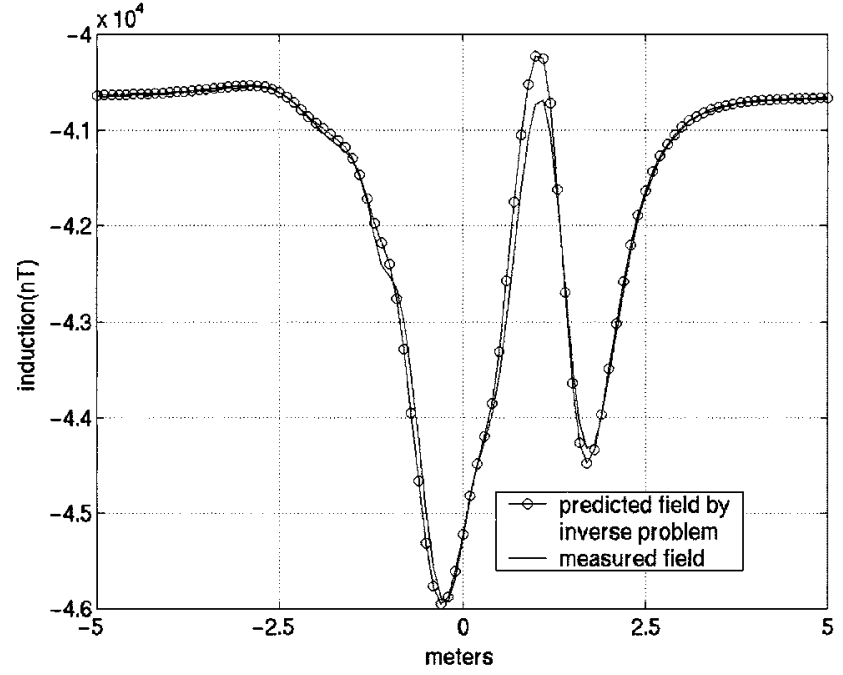

Fig. 5. Comparison of field predicted by inverse problem and measured field. Vertical component of the induction outside the ship $(30 \mathrm{~cm}$ under the hull)

\section{CONCLUSION}

We have developed a new method to solve magnetostatic inverse problem and tested it on real measurements. It presents advantages in comparison to classical regularization. First, we reduced the order of the singularity of (5), which allows to place sensors closer to the sources (i.e., the shell) and to obtain more accurate measurements. Second, by adding an internal physical system for the shell, we improved the problem analysis. Fewer measurements (so, fewer sensors) lead to a reliable solution and systems obtained have generally a good condition number. Neither regularization nor choice of weighted parameter is needed (the choice of this parameter is a real difficulty for 3-D shell devices). We think that instead of trying to solve ill-posed problem by mathematical analysis, it is better to solve a "well-posed" one. Moreover, our approach can easily be applied to volume devices and is not restricted to boundary element formulations. A future work will deal with its implementation with a finite element method in FLUX3-D software.

\section{REFERENCES}

[1] X. Brunotte and G. Meunier, "Line element for efficient computation of the magnetic field created by thin iron plates," IEEE Trans. Magn., vol. 26, pp. 2196-2198, Sept. 1990.

[2] L. Krahenbuhl and D. Muller, "Thin layer in electrical engineering. Example of shell models in analyzing eddy-currents by boundary and finite element methods," IEEE Trans. Magn., vol. 29, pp. 1450-1455, Mar. 1993.

[3] O. Chadebec, J.-L. Coulomb, V. Leconte, J. P. Bongiraud, and G. Cauffet, "Modeling of static magnetic anomaly created by iron plates," IEEE Trans. Magn., vol. 36, pp. 667-671, Oct. 2000.

[4] H. Igarashi, T. Honma, and A. Kost, "Inverse inference of magnetization distribution in cylindrical permanent magnets," IEEE Trans. Magn., vol. 36, pp. 1168-1171, July 2000.

[5] A. N. Tikhonov and V. Y. Arsenine, Solution of Ill-Posed Problem. Washington, DC: Wiston/Wiley, 1977.

[6] P. C. Hansen and D. P. O'leary, "The use of the L-curve in the regularization of discretization of discrete ill-posed problem," SIAM, J. Sci. Comput. 14, pp. 1487-1503, 1993. 